\title{
The relationship between cancer and medication exposure in patients with systemic lupus erythematosus: a nested case-control study
}

\author{
Jinyan Guo ${ }^{1}$, Zhigang Ren², Jianhao Li ${ }^{2}$, Tianfang Li ${ }^{1}$, Shengyun Liu ${ }^{1 *}$ and Zujiang $\mathrm{Yu}^{2^{*}}$
}

\begin{abstract}
Background: Systemic lupus erythematosus (SLE) is associated with increased risk of cancer and the mechanism remains unclear. Here, we examined the level of auto-antibodies and disease activity index scores in SLE patients with cancers and analyzed whether medications for SLE management might contribute to the higher cancer risk in SLE patients.

Methods: In this retrospective study, we carried out a nested case-control study in a large cohort of SLE patients. We screened 5858 SLE patients to identify the newly diagnosed and yet to be treated cancers. The following clinical features were evaluated: auto-antibodies levels, SLE disease activity index scores, and previous medication used for SLE management. Systemic glucocorticoid, cyclophosphamide, hydroxychloroquine (HCQ), methotrexate, and azathioprine were considered the main medication indices.

Results: Our analyses identified 51 SLE patients who also had cancer and 204 matched control patients who had SLE but not cancer. Of the 51 SLE patients, thyroid cancer (14/51, 27.45\%), cervical cancer (10/51, 19.61\%), and lung cancer $(7 / 51,13.73 \%)$ were the most common types. Our analyses did not reveal any significant differences in the levels of auto-antibodies in SLE patients with cancers relative to the control group. Further, we observed that disease activity was significantly lower in SLE patients with cancers relative to the matched control SLE group. There was no statistically significant association between the cancer risk and the use of systemic glucocorticoid, cyclophosphamide, methotrexate, or azathioprine. Importantly, the administration of HCQ was significantly lower in SLE patients suffering cancers relative to the cancer-free matched control group.

Conclusions: Our analyses indicate that SLE patients with cancers might have a lower disease activity at the time of cancer diagnosis. HCQ was negatively associated with cancer risk in SLE patients. These findings highlight a potential and novel prevention strategy for SLE.
\end{abstract}

Keywords: Lupus, Cancer, Autoantibody, Disease activity, Hydroxychloroquine

\footnotetext{
* Correspondence: fccliusy@zzu.edu.cn; johnyuem@zzu.edu.cn

'Department of Rheumatology and Immunology, The First Affiliated Hospital

of Zhengzhou University, No. 1, Jianshe East Road, Zhengzhou 450052,

Henan Province, China

${ }^{2}$ Department of Infectious Disease, The First Affiliated Hospital of Zhengzhou University, No. 1, Jianshe East Road, Zhengzhou 450052, Henan Province, China
}

C C The Author(s). 2020 Open Access This article is licensed under a Creative Commons Attribution 4.0 International License, which permits use, sharing, adaptation, distribution and reproduction in any medium or format, as long as you give appropriate credit to the original author(s) and the source, provide a link to the Creative Commons licence, and indicate if changes were made. The images or other third party material in this article are included in the article's Creative Commons licence, unless indicated otherwise in a credit line to the material. If material is not included in the article's Creative Commons licence and your intended use is not permitted by statutory regulation or exceeds the permitted use, you will need to obtain permission directly from the copyright holder. To view a copy of this licence, visit http://creativecommons.org/licenses/by/4.0/ The Creative Commons Public Domain Dedication waiver (http://creativecommons.org/publicdomain/zero/1.0/) applies to the data made available in this article, unless otherwise stated in a credit line to the data. 


\section{Background}

Systemic lupus erythematosus (SLE) is an autoimmune inflammatory disorder characterized by an aberrant production of auto-antibodies and a wide range of clinical manifestations and complications. Antinuclear antibodies (ANAs) refer to a broad class of antibodies targeting a wide range of cellular and nuclear components. These class of antibodies are generated as a result of loss of immune tolerance. Anti-double-stranded DNA antibody (anti-dsDNA) and anti-Sm antibody (anti-Sm) are the important hallmarks of SLE [1]. For patients presenting with SLE, treatment with hydroxychloroquine (HCQ) is recommended unless contraindicated. In cases where the disease affects major organs or present refractory symptoms, treatment with systemic glucocorticoid (GC), cyclophosphamide (CTX), methotrexate (MTX), or azathioprine (AZA) is recommended. As early diagnosis and advanced treatments have significantly improved the survival, malignancies are becoming an important cause of mortality in SLE patients [2-10]. However, the mechanism underlying such an increase in cancer risk is not completely understood.

Although they are important serological markers of autoimmune disease, ANAs are not unique to autoimmune disorders and multiple studies have reported the involvement of ANAs in a variety of neoplastic diseases $[11,12]$. Interestingly, several lines of evidence suggested that ANAs have anti-neoplastic effects in cancer patients without concomitant autoimmune diseases [13, 14] and are associated with a better prognosis [15-17]. A previous report showed that the damage index, defined by the Systemic Lupus International Collaborating Clinics/American College of Rheumatology (SLICC/ACR), is associated with overall cancer risk [18]. Contradictory findings were observed in another study in which no association was found between the adjusted mean SLE Disease Activity Index 2000 (SLEDAI-2 K) and the risk of lymphoma [19]. Similarly, the relationship between the use of immunosuppressant and cancer risk in SLE is inconsistent. A previous report showed that the application of CTX and AZA did not increase cancer risk in SLE patients [20]. Another study demonstrated that although immunosuppressants including CTX, AZA, and MTX were not associated with overall cancer risk, they may increase the risk of hematological malignancies in patients with SLE [18]. It has been reported that CTX administration is associated with increased cancer risk while administration of HCQ is thought to lower cancer risk [21].

To improve our understanding of the relationship between cancer risk and SLE pharmacologic interventions, we carried out a nested case-control study. To this end, we analyzed clinical features including auto-antibodies and disease activity in 5858 SLE patients as well as the pharmacologic interventions used in the management of
SLE. Our results showed that SLE patients with cancers had lower disease activity and that HCQ was negatively associated with cancer risk in these patients.

\section{Methods \\ Study design}

All patients recruited into this retrospective cohort study met the updated American College of Rheumatology criteria for the classification of SLE [22]. All patients included in the study were hospitalized at the First Affiliated Hospital of Zhengzhou University between October 1, 2010, and October 1, 2019. Any SLE patients younger than 18 years old or less than 18 years at SLE diagnosis age were excluded from the study. Cancer diagnosis was confirmed by histological analyses, and any patients diagnosed with premalignant lesions were excluded. Patients with a concomitant diagnosis of rheumatoid arthritis, SjÖgren syndrome, inflammatory myopathy, autoimmune hepatitis, or primary biliary cholangitis were defined as having overlap syndrome. Participants were assigned into a cancer group and control group. This study was approved by the Ethical Committee of the First Affiliated Hospital of Zhengzhou University, ethical approval number no.2019-KY-199 (Additional file 1).

\section{Clinical and laboratory examinations}

The following patient information was collected: age, gender, age at SLE diagnosis, and course of SLE progression as well as chronic comorbidities including hypertension, diabetes mellitus, and dyslipidemia. In addition, the positive rate and titers of autoantibodies were assessed including antinuclear antibody (ANA) and other autoantibodies such as anti-dsDNA, anti-Sm, antiRo52 antibody (anti-Ro52), anti-Ro60 antibody (antiRo60), anti-SSB antibody (anti-SSB), anti-nucleosome antibody (anti-Nuc), anti-histone antibody (anti-His), anti-ribosome antibody (anti-Rib), and anti-nRNP antibody (anti-nRNP). The SLE Disease Activity Index (SLEDAI) scores were calculated at the day of cancer diagnosis as previously described [23]. The information on the use of GC, HCQ, CTX, MTX, and AZA was also collected from the date of SLE diagnosis to the date of cancer diagnosis for participants in the cancer group or the date of admission for those in the control group.

\section{Propensity score matching}

Propensity score matching was performed to minimize selection bias when evaluating the effect of immunosuppressant on cancer risk. Based on the propensity scores, each participant with cancer was matched with four cancer-free participants. The propensity score was calculated by taking into account the following variables: age, gender, age at SLE diagnosis, disease course of SLE and 
comorbidities such as hypertension, diabetes mellitus, and dyslipidemia.

\section{Statistical analysis}

Patient clinical features and SLEDAI scores were compared between the cancer group and the control group using an independent-sample $t$ test for continuous variables or the chi-square test for categorical variables. Conditional logistic regression analysis was used for the evaluation of the association between cancer odds and medical intervention with pharmacologic agents. Cancer occurrence was treated as a dependent variable in the logistic analysis. Associations were firstly evaluated without consideration for confounding factors followed by an analysis taking into account such factors (Table 1). SPSS statistical software version 20.0 was used to conduct data analysis and propensity score matching [SPSS Inc., Chicago, IL].

\section{Results}

\section{Patient characteristics}

A total of 5858 patients diagnosed with SLE between October 1, 2010, and October 1, 2019, were recruited into this study. Eighteen patients that had been diagnosed with cancer prior to SLE diagnosis, 18 patients that had metastasis or received chemotherapy prior to hospital admission, and 274 patients with overlap syndrome were excluded from subsequent analyses. Of the 5548 patients that were eligible for further analyses, 51 were cancer patients while the remaining 5497 were cancer-free patients. Each cancer case was matched with four cancer-free patients. Our study therefore consisted of 51 cancer patients and 204 matched cancer-free patients (Fig. 1).

Patients' characteristics of the cancer group and the control group are showed in Table 1. Before matching, patients in the cancer group were older, diagnosed with SLE at a more advanced age, and had a longer disease course of SLE and a higher prevalence of comorbidities. However, such difference was not clear after matching (Table 1).

\section{Distribution of all cancers and specific cancer types}

The specific types of cancer are showed in Table 2. Four patients had hematological cancer (2 leukemia and 2 non-Hodgkin's lymphoma). No patient had Hodgkin's lymphoma in this cohort. A total of 47 SLE patients had non-hematological cancer, with thyroid cancer being the most frequently observed type of cancer (27.45\%), followed by cervical cancer $(19.61 \%)$ and lung cancer (13.73\%).

Level of auto-antibodies in the cancer and control groups The levels of auto-antibodies in both groups are summarized in Table 3. No significant differences were observed between the two groups in the positive rate of ANA, anti-dsDNA, anti-Sm, anti-RO52, anti-RO60, antiSSB, anti-Nuc, anti-His, anti-Rib, and anti-nRNP.

\section{SLEDAI and disease activity indexes in cancer and control groups}

The SLEDAI and disease activity indexes in cancer and control groups are showed in Table 4. Patients in the control group always had a higher percentage of decreased C3 and elevated proteinuria than in the cancer group $(82.22 \%$ vs $44.44 \%, P<0.01 ; 36.00 \%$ vs $12.24 \%$, $P<0.01$; respectively). The SLEDAI was higher in the control group than that in the cancer group ( 8 vs $2, P<$ 0.01 ). No significant differences in low $C 4$, low white blood cell, and thrombocytopenia were observed between the two groups.

\section{Medication exposure and cancer risk}

The results of association analysis between medication exposure and cancer odds are provided in Fig. 2 and Additional file 2.

Univariate analysis revealed that HCQ was associated with a lower odds of cancer $(\mathrm{OR}=0.417, \mathrm{CI} 0.220$, $0.791)$, while $\mathrm{GC}(\mathrm{OR}=0.783$, CI $0.273,2.248)$, CTX $(\mathrm{OR}=1.378$, CI 0.517, 3.670), $\mathrm{MTX} \quad(\mathrm{OR}=0.788$, CI $0.219,2.831)$, and AZA (OR $=0.653$, CI 0.141, 3.014) was not significantly associated with cancer odds. The

Table 1 Characteristics of patients in the cancer and control groups

\begin{tabular}{|c|c|c|c|c|c|c|}
\hline \multirow[t]{2}{*}{ Characteristics } & \multicolumn{3}{|l|}{ Before matching } & \multicolumn{3}{|l|}{ After matching } \\
\hline & Cancer group $(n=51)$ & Control group $(n=5497)$ & $P$ value & Cancer group $(n=51)$ & Control group $(n=204)$ & $P$ value \\
\hline Age, median & 47 & 35 & $<0.0001$ & 47 & 46 & 0.69 \\
\hline Female gender, $n(\%)$ & $49,96.08$ & $4930,89.68$ & 0.2055 & $49,96.08$ & $192,94.12$ & 0.74 \\
\hline Age at SLE diagnosis, median & 41 & 33 & 0.0024 & 41 & 39 & 0.96 \\
\hline Disease course of SLE, median & 60 & 6 & $<0.0001$ & 60 & 60 & 0.92 \\
\hline Hypertension & $5,9.80 \%$ & $199,3.620 \%$ & 0.0195 & $5,9.80 \%$ & $15,7.35 \%$ & 0.56 \\
\hline Diabetes mellitus & $5,9.80 \%$ & $189,3.438 \%$ & 0.0375 & $5,9.80 \%$ & $14,6.86 \%$ & 0.55 \\
\hline Dyslipidemia & $8,15.38 \%$ & $494,8.18 \%$ & 0.0969 & $8,15.38 \%$ & $28,13.72 \%$ & 0.72 \\
\hline
\end{tabular}




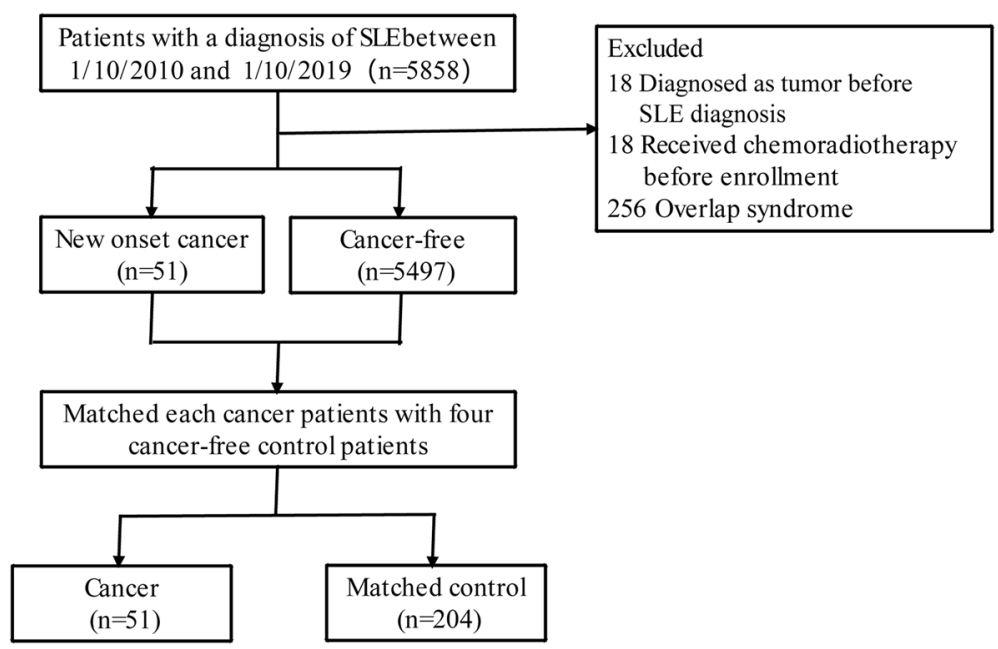

Fig. 1 Flow chart of the study design

results were not changed after adjustment for confounding variables.

\section{Discussion}

Numerous studies have demonstrated that patients with SLE had an increased overall cancer risk compared with the general healthy age and sex matched population, especially non-Hodgkin's lymphoma, thyroid cancer, lung

Table 2 Specific types of cancers in the cancer cohort

\begin{tabular}{ll}
\hline Sites and types & $N(\%)$ \\
\hline Hematological cancer & $2(3.92 \%)$ \\
Leukemia & $2(3.92 \%)$ \\
Non-Hodgkin's lymphoma & \\
Non-hematological cancer & \\
Reproductive system & $10(19.61 \%)$ \\
Cervical cancer & $2(3.92 \%)$ \\
Vulvar cancer & $1(1.96 \%)$ \\
Uterus cancer & \\
Non-reproductive system & $14(27.45 \%)$ \\
Thyroid cancer & $7(13.73 \%)$ \\
Lung cancer & $3(5.89 \%)$ \\
Gastric carcinoma & $2(3.92 \%)$ \\
Rectal carcinoma & $1(1.96 \%)$ \\
Hepatic carcinoma & $1(1.96 \%)$ \\
Appendix cancer & $1(1.96 \%)$ \\
Bile duct cancer & $1(1.96 \%)$ \\
Pancreatic cancer & $2(3.92 \%)$ \\
Renal cell cancer & $2(3.92 \%)$ \\
Breast cancer &
\end{tabular}

cancer, and vulva cancer. The mechanism remains unclear, it is speculated that various factors including medication exposure, the activated auto-immune system, viral infection, and overlap syndrome as well as traditional lifestyle cancer risk factors may all contribute to the increased cancer risk in SLE $[6,7]$. To the best of our knowledge, only a handful of studies have been done to explore the association between cancer and the drugs used in SLE, and the results were inconsistent [18-21]. In this large nested case-control study, we found that the SLE patients with cancer had lower disease activity and that HCQ was negatively associated with cancer risk in SLE patients.

Both SLE and cancer have been associated with immune dysfunction [24]. In SLE patients, the impaired immune system is not able to discriminate between self and non-self-antigens, leading to aberrant production of autoantibodies causing host tissue damage. On the contrary, cancer formation is caused by compromised host's immune system that cannot recognize cancer antigens. It has been previously reported that the immunogenicity of cancer cell could induce the production of a wide range of auto-antibodies including ANA, anti-dsDNA, anti-Sm, anti-SSA, anti-SSB, anti-Rib, and anti-nRNP [25]. The level of ANA has been reported to be elevated in $31.5 \%$ lymphoma patients relative to the control group [12]. While it is well established that anti-dsDNA is highly specific for SLE, it has been found in patients with different malignancies and may serve as a prognostic indicator for cancer. The association between antidsDNA and cancer was firstly demonstrated in bronchogenic carcinoma [26]. One study suggested that this antibody may play a role in the pathogenesis of lymphoma and thymoma [27]. It has been hypothesized that the presence of anti-dsDNA autoantibodies in patients 
Table 3 Comparison of levels of auto-antibodies between the two groups

\begin{tabular}{llll}
\hline Auto-antibodies & Cancer group $(n=51)($ positive/total, percentage) & Control group $(n=204)$ (positive/total, percentage) & $P$ value \\
\hline ANA & $37 / 37,100 \%$ & $198 / 200,99.00 \%$ & 1.00 \\
Anti-dsDNA & $15 / 35,42.86 \%$ & $95 / 180,52.78 \%$ & 0.36 \\
Anti-Sm & $5 / 36,13.89 \%$ & $33 / 172,19.19 \%$ & 0.63 \\
Anti-RO52 & $26 / 37,70.27 \%$ & $110 / 172,63.95 \%$ & 0.57 \\
Anti-RO60 & $20 / 37,54.05 \%$ & $110 / 172,63.95 \%$ & 0.57 \\
Anti-SSB & $4 / 36,11.11 \%$ & $16 / 172,9.30 \%$ & 0.76 \\
Anti-Nuc & $11 / 36,30.56 \%$ & $70 / 172,40.70 \%$ & 0.35 \\
Anti-His & $7 / 36,19.44 \%$ & $50 / 172,29.07 \%$ & 0.31 \\
Anti-Rib & $10 / 36,27.78 \%$ & $58 / 172,33.72 \%$ & 0.56 \\
Anti-nRNP & $11 / 36,30.56 \%$ & $64 / 172,37.21 \%$ & 0.57
\end{tabular}

ANA antinuclear antibody, anti-dsDNA anti-double-stranded DNA antibody, anti-Sm anti-Sm antibody, anti-RO52 anti-RO52 antibody, anti-RO60 anti-RO60 antibody, anti-SSB anti-SSB antibody, anti-NuC anti-nucleosome antibody, anti-His anti-histone antibody, anti-Rib anti-ribosome antibody, anti-nRNP anti-nRNP antibody

with colorectal cancer might indicate better disease outcome [15]. Our current study evaluated the significance of disparities in the levels of ANA, anti-dsDNA, anti-Sm, anti-RO52, anti-RO60, anti-SSB, anti-Nuc, anti-His, anti-Rib, and anti-nRNP antibodies between the cancer group and the cancer-free control group at the time of cancer diagnosis. Our results demonstrated there was no significant difference in the levels of these factors between the cancer group and the control group at the time of cancer diagnosis.

The organ damage and disease activity in SLE patients with or without cancers were investigated, and the results were inconsistent, likely due to the differences in inclusion criteria, race, and scoring systems. For instance, a mean SLICC/ACR damage score of 1.9 and 1.7 has been reported for the cancer group and the control group respectively, suggesting that organ injury was more severe in the cancer group [18]. A different study did not find statistically significant differences in the adjusted mean SLEDAI-2 K between a lymphoma group and a control group [19]. It should be noted that while SLICC/ACR mainly evaluates organ damage [28], the adjusted mean SLEDAI-2 K reflects the mean disease activity after onset [29]. Our results indicate that SLE with different malignancies had lower SLEDAI scores, lower rates of renal involvement, and low level of complement compared with the control group. The SLEDAI mainly reflects the disease activity within 10 days [23]. Taken together, these data indicate that SLE patients with cancers have lower disease activity at the time of cancer diagnosis.

The role of immunosuppressant in cancer development in SLE patients remains controversial. One study showed that immunosuppressant therapy was not associated with overall cancer risk in patients with SLE but might contribute to an increased risk of hematological malignancy [18]. A different research reported that exposure to CTX might contribute to a higher lymphoma risk in SLE patients [19], although this was contradicted by a different report showing that the use of CTX and AZA did not contribute to lymphoma risk [20]. It has been demonstrated that CTX increases cancer risk in SLE patients in a dose-dependent manner [21]. Therefore, more investigations looking at a larger number of participants are needed. In fact, numerous studies have demonstrated that the activated auto-immune system may contribute to the increased cancer risk in patients with SLE, especially non-Hodgkin's lymphoma. The probable mechanism is the detective immune surveillance system. By virtue of the disease, SLE patients have impaired immune surveillance system due to the activated auto-immune system. In healthy immune system, aberrant cells produced during cell replication are

Table 4 Comparison of SLEDAl between the two groups

\begin{tabular}{llll}
\hline Indicator & Cancer group $(n=51)$ (positive/total, percentage) & Control group $(n=204)$ (positive/total, percentage) & $P$ value \\
\hline Low C3 & $16 / 36,44.44 \%$ & $74 / 90,82.22 \%$ & $<0.01$ \\
Low C4 & $14 / 36,38.89 \%$ & $48 / 90,53.33 \%$ & 0.17 \\
Low WBC & $6 / 48,12.50 \%$ & $26 / 102,25.49 \%$ & 0.09 \\
Low PLT & $10 / 48,20.83 \%$ & $34 / 102,33.33 \%$ & 0.13 \\
Proteinuria & $6 / 49,12.24 \%$ & $36 / 100,36.00 \%$ & $<0.01$ \\
SLEDAl, median & 2 & 8 & $<0.01$ \\
\hline
\end{tabular}




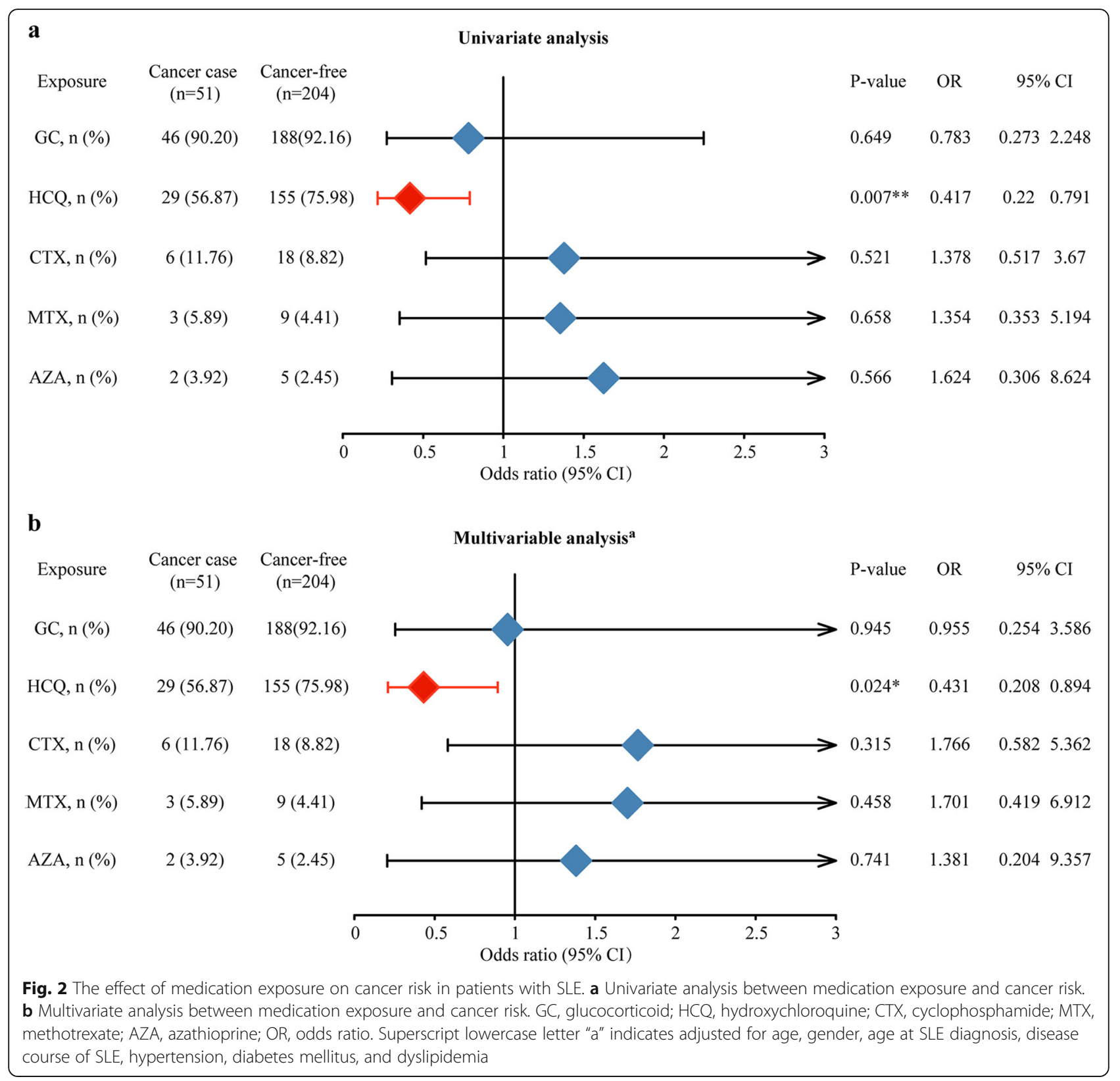

eliminated to prevent them from becoming malignant. In SLE patients, this regulation process may be impaired, making patients more vulnerable to develop cancers. At the same time, the abnormal apoptotic process inherent in SLE may enhance this process $[24,30]$. In the scenario of lymphomas occurring in SLE patients, besides the mechanism mentioned above, there is a further aspect that has to be taken into account: these malignancies arise from the immune system itself. The activated lymphocytes in SLE are prone to potentially dangerous genetic events during their maturation, such as recombination or hypermutation in B cells, which eventually promote the development of lymphoma, particularly non-Hodgkin's lymphoma [31, 32].
HCQ is extensively used in SLE treatment. Besides its well-established effects on the skin and joint symptoms, several studies have indicate that HCQ has important longterm effects on lupus, including reduced long-term accrual damage and decreased long-term mortality [33, 34]. A protective function of antimalarial against cancer in SLE patients has been proposed [35]. Hsu et al. found that HCQ decreased cancer risk in a dose-dependent manner [21]. Our current large-scale study has also elucidated a negative association between HCQ and cancer.

It has been proposed that HCQ might modulate autophagy by impacting lysosomal acidification and blocking the fusion of auto-phagosomes with lysosomes [36]. Chloroquine may trigger the expression of Tp53 which 
may protect the cells from genotoxic stimuli [37]. In addition, the antimalarial may inhibit unlimited replication of cancer cells via their strong DNA intercalating properties [38]. Chloroquine may promote DNA repair following DNA damage as a result of alkylating therapy [39]. Multiple preclinical and clinical trials have demonstrated a synergistic anticancer effect of HCQ with chemotherapies and targeted therapies [40]. For instance, cytotoxicity of tamoxifen against breast cancer cells has been shown to be enhanced by combination therapy with HCQ [41]. In addition, HCQ is effective against hepatocellular carcinoma and pancreatic ductal adenocarcinoma [42, 43], as well as hematologic cancers like chronic myeloid leukemia, myeloma, and lymphoma [44-46]. Taken together, these reports suggest that HCQ may decrease the cancer risk in SLE patients.

In the SLE cohort included in this study, thyroid cancer, cervical cancer, and lung cancer were the top three cancer types. Studies suggest an increased risk of cervical cancer among SLE patients compared with the general population $[47,48]$. It has been reported that immunosuppressant increases the risk of cervical neoplasia in SLE patients and this is attributable to decreased HPV clearance $[47,49]$. This suggests that SLE patients under immunosuppressive agents should undergo regular screening for cervical dysplasia.

This retrospective study of a large cohort of SLE patients examined the odds of being diagnosed with cancer in SLE patients. Our results suggest that SLE patients with cancers have lower disease activity at the time of cancer diagnosis. In addition, a negative association between HCQ administration and cancer risk in SLE patients was unveiled, highlighting a novel potential cancer prevention strategy for SLE patients.

\section{Conclusions}

Our analyses indicate that SLE patients with cancers might have a lower disease activity at the time of cancer diagnosis. HCQ was negatively associated with cancer risk in SLE patients. These findings highlight a potential and novel prevention strategy for SLE.

\section{Supplementary information}

Supplementary information accompanies this paper at https://doi.org/10. 1186/s13075-020-02228-6.

Additional file 1. Ethical approval certification of this study.

Additional file 2. Association between medication exposure and cancer risk.

\section{Abbreviations}

SLE: Systemic lupus erythematosus; HCQ: Hydroxychloroquine; ANAs: Antinuclear antibodies; anti-dsDNA: Anti-double-stranded DNA antibody; anti-Sm: Anti-Sm antibody; GC: Systemic glucocorticoid; CTX: Cyclophosphamide; MTX: Methotrexate; AZA: Azathioprine; SLICC/ ACR: Systemic Lupus International Collaborating Clinics/American College of
Rheumatology; SLEDAI-2 K: SLE Disease Activity Index 2000; ANA: Antinuclear antibody; anti-Ro52: Anti-Ro52 antibody; anti-Ro60: Anti-Ro60 antibody; antiSSB: Anti-SSB antibody; anti-Nuc: Anti-nucleosome antibody; anti-His: Antihistone antibody; anti-Rib: Anti-ribosome antibody; anti-nRNP: Anti-nRNP antibody; SLEDAl: SLE Disease Activity Index; WBC: White blood cell; PLT: Platelet; OR: Odds ratio

\section{Acknowledgements}

We thank the many clinical doctors from the Oncology Department and Information Department, First Affiliated Hospital of Zhengzhou University, who were involved in this study.

\section{Authors' contributions}

$J G, Z R, S L$, and ZY designed the study. JG and JL collected clinical data. JG, $Z R$, and $J L$ analyzed the data. JG, ZR, and TL wrote the manuscript. All authors reviewed and approved the manuscript.

\section{Funding}

The study was supported by the National S\&T Major Project of China (2018ZX10301201-008), Key Scientific Research Projects of Higher Education Institutions in Henan Province (20A320056), National Key Research and Development Program of China (2018YFC2000501), and China Postdoctoral Science Foundation (2017 M610463).

\section{Availability of data and materials}

The dataset analyzed in this paper is available from the corresponding author on reasonable request, and with appropriate additional ethical approvals, where necessary.

Ethics approval and consent to participate

This study was approved by the Ethical Committee of the First Affiliated Hospital of Zhengzhou University, ethical approval number no.2019-KY-199.

\section{Consent for publication}

All the results presented in this article are in aggregate form, and no personally identifiable information was used for this study.

\section{Competing interests}

The authors declare that they have no competing interests.

Received: 16 January 2020 Accepted: 2 June 2020

Published online: 26 June 2020

References

1. Rekvig OP, Putterman C, Casu C, et al. Autoantibodies in lupus: culprits or passive bystanders? Autoimmun Rev. 2012:11(8):596-603.

2. Bernatsky $S$, Boivin JF, Joseph $L$, et al. An international cohort study of cancer in systemic lupus erythematosus. Arthritis Rheum. 2005;52(5):1481-90.

3. Pettersson T, Pukkala $E$, Teppo $L$, et al. Increased risk of cancer in patients with systemic lupus erythematosus. Ann Rheum Dis. 1992;51(4):437-9.

4. Björnådal L, Löfström B, Yin L, et al. Increased cancer incidence in a Swedish cohort of patients with systemic lupus erythematosus. Scand J Rheumatol. 2002;31(2):66-71.

5. Mellemkjaer L, Andersen V, Linet MS, et al. Non-Hodgkin's lymphoma and other cancers among a cohort of patients with systemic lupus erythematosus. Arthritis Rheum. 1997:40(4):761-8.

6. Bernatsky S, Ramsey-Goldman R, Labrecque J, et al. Cancer risk in systemic lupus: an updated international multi-centre cohort study. J Autoimmun. 2013:42:130-5.

7. Chen YJ, Chang YT, Wang CB, et al. Malignancy in systemic lupus erythematosus: a nationwide cohort study in Taiwan. Am J Med. 2010; 123(12):1150.e1151-6.

8. Moss KE, loannou Y, Sultan SM, et al. Outcome of a cohort of 300 patients with systemic lupus erythematosus attending a dedicated clinic for over two decades. Ann Rheum Dis. 2002;61(5):409-13.

9. Ruiz-Irastorza G, Egurbide MV, Pijoan Jl, et al. Effect of antimalarials on thrombosis and survival in patients with systemic lupus erythematosus. Lupus. 2006;15(9):577-83.

10. Bernatsky S, Ramsey-Goldman R, Clarke A. Exploring the links between systemic lupus erythematosus and cancer. Rheum Dis Clin North Am. 2005; 31(2):387-402, viii-ix. 
11. Imran A, Neelam F, Tariq M. Incidence of circulating antinuclear antibodies in cancer patients. Indian J Med Sci. 2003;57(3):113-6.

12. Zou HY, Gu X, Yu WZ, et al. Detection of serum antinuclear antibodies in lymphoma patients. Genet Mol Res. 2015;14(4):16546-52.

13. Torchilin VP, lakoubov LZ, Estrov Z. Antinuclear autoantibodies as potential antineoplastic agents. Trends Immunol. 2001:22(8):424-7.

14. Lv S, Zhang J, Wu J, et al. Origin and anti-tumor effects of anti-dsDNA autoantibodies in cancer patients and tumor-bearing mice. Immunol Lett. 2005;99(2):217-27.

15. Syrigos KN, Charalambopoulos A, Pliarchopoulou K, et al. The prognostic significance of autoantibodies against dsDNA in patients with colorectal adenocarcinoma. Anticancer Res. 2000;20:4351-3.

16. Mitchell $P$, Thatcher N, Socinski MA, et al. Tecemotide in unresectable stage III non-small-cell lung cancer in the phase III START study: updated overall survival and biomarker analyses. Ann Oncol. 2015;26(6):1134-42.

17. Maire C, Vercambre-Darras S, Devos P, et al. Metastatic melanoma: spontaneous occurrence of auto antibodies is a good prognosis factor in a prospective cohort. J Eur Acad Dermatol Venereol. 2013;27(1):92-6.

18. Bernatsky $S$, Joseph $L$, Boivin JF, et al. The relationship between cancer and medication exposures in systemic lupus erythaematosus: a case-cohort study. Ann Rheum Dis. 2008:67(1):74-9.

19. Bernatsky S, Ramsey-Goldman R, Joseph L, et al. Lymphoma risk in systemic lupus: effects of disease activity versus treatment. Ann Rheum Dis. 2014; 73(1):138-42.

20. Löfström B, Backlin C, Sundström C, et al. A closer look at non-Hodgkin's lymphoma cases in a national Swedish systemic lupus erythematosus cohort: a nested case-control study. Ann Rheum Dis. 2007;66(12):1627-32.

21. Hsu CY, Lin MS, SuYJ, et al. Cumulative immunosuppressant exposure is associated with diversified cancer risk among 14832 patients with systemic lupus erythematosus: a nested case-control study. Rheumatology (Oxford). 2017:56(4):620-8.

22. Hochberg MC. Updating the American College of Rheumatology revised criteria for the classification of systemic lupus erythematosus. Arthritis Rheum. 1997:40(9):1725.

23. Bombardier C, Gladman DD, Urowitz MB, et al. Derivation of the SLEDAI. A disease activity index for lupus patients. The Committee on Prognosis Studies in SLE. Arthritis Rheum. 1992;35(6):630-40.

24. Singh G, Agarwal AK, Prosek J, et al. Can killers be saviors? Lupus. 2017;26(9): 903-8.

25. Vlagea A, Falagan S, Gutiérrez-Gutiérrez G, et al. Antinuclear antibodies and cancer: a literature review. Crit Rev Oncol Hematol. 2018;127:42-9.

26. Dellen $\mathrm{H}$, Seemayer $\mathrm{NH}$. Autoimmunity in patients with bronchogenic carcinomas: occurrence of antibodies against nuclear antigens, chromosomes and ds-deoxyribonucleic acid. Zentralbl Bakteriol Mikrobiol Hyg B. 1983;177(6):451-68.

27. Attar SM, Koshak EA. Medical conditions associated with a positive antidouble-stranded deoxyribonucleic acid. Saudi Med J. 2010;31(7):781-7.

28. Gladman D, Ginzler E, Goldsmith C, et al. The development and initial validation of the Systemic Lupus International Collaborating Clinics/ American College of Rheumatology damage index for systemic lupus erythematosus. Arthritis Rheum. 1996;39(3):363-9.

29. Ibañez D, Urowitz MB, Gladman DD. Summarizing disease features over time: I. Adjusted mean SLEDAI derivation and application to an index of disease activity in lupus. J Rheumatol. 2003;30(9):1977-82.

30. Gayed M, Bernatsky S, Ramsey-Goldman R, et al. Lupus and cancer. Lupus. 2009;18(6):479-85.

31. Klein A, Polliack A, Gafter-Gvili A. Systemic lupus erythematosus and lymphoma: incidence, pathogenesis and biology. Leuk Res. 2018;75:45-9.

32. Schmalzing $M$. The relationship of lymphoma and lupus-at least bidirectional. Rheumatology. 2019;58(7):1131-2.

33. Alarcón GS, McGwin G, Bertoli AM, et al. Effect of hydroxychloroquine on the survival of patients with systemic lupus erythematosus: data from LUMINA, a multiethnic US cohort (LUMINA L). Ann Rheum Dis. 2007:66(9): 1168-72.

34. Petri $M$, Purvey $S$, Fang $H$, et al. Predictors of organ damage in systemic lupus erythematosus: the Hopkins Lupus Cohort. Arthritis Rheum. 2012; 64(12):4021-8.

35. Ruiz-Irastorza G, Ugarte A, Egurbide MV, et al. Antimalarials may influence the risk of malignancy in systemic lupus erythematosus. Ann Rheum Dis. 2007:66(6):815-7.
36. White E. Deconvoluting the context-dependent role for autophagy in cancer. Nat Rev Cancer. 2012;12(6):401-10.

37. Sohn TA, Bansal R, Su GH, et al. High-throughput measurement of the Tp53 response to anticancer drugs and random compounds using a stably integrated Tp53-responsive luciferase reporter. Carcinogenesis. 2002;23(6): 949-57.

38. Sotelo J, Briceño E, López-González MA. Adding chloroquine to conventional treatment for glioblastoma multiforme: a randomized, doubleblind, placebo-controlled trial. Ann Intern Med. 2006;144(5):337-43.

39. Briceño E, Reyes S, Sotelo J. Therapy of glioblastoma multiforme improved by the antimutagenic chloroquine. Neurosurg Focus. 2003;14(2):e3.

40. Amaravadi RK, Lippincott-Schwartz J, Yin XM, et al. Principles and current strategies for targeting autophagy for cancer treatment. Clin Cancer Res. 2011;17(4):654-66

41. Cook KL, Wärri A, Soto-Pantoja DR, et al. Hydroxychloroquine inhibits autophagy to potentiate antiestrogen responsiveness in ER+ breast cancer. Clin Cancer Res. 2014;20(12):3222-32.

42. Li J, Yang B, Zhou Q, et al. Autophagy promotes hepatocellular carcinoma cell invasion through activation of epithelial-mesenchymal transition. Carcinogenesis. 2013;34(6):1343-51.

43. Yang A, Kimmelman AC. Inhibition of autophagy attenuates pancreatic cancer growth independent of TP53/TRP53 status. Autophagy. 2014;10(9): 1683-4.

44. Helgason GV, Mukhopadhyay A, Karvela M, et al. Autophagy in chronic myeloid leukaemia: stem cell survival and implication in therapy. Curr Cancer Drug Targets. 2013;13(7):724-34

45. Pan Y, Gao Y, Chen $L$, et al. Targeting autophagy augments in vitro and in vivo antimyeloma activity of DNA-damaging chemotherapy. Clin Cancer Res. 2011;17(10):3248-58.

46. Yang ZJ, Chee CE, Huang S, et al. The role of autophagy in cancer: therapeutic implications. Mol Cancer Ther. 2011;10(9):1533-41.

47. Wadström H, Arkema EV, Sjöwall C, et al. Cervical neoplasia in systemic lupus erythematosus: a nationwide study. Rheumatology (Oxford). 2017; 56(4):613-9

48. Zard E, Arnaud L, Mathian A, et al. Increased risk of high grade cervical squamous intraepithelial lesions in systemic lupus erythematosus: a metaanalysis of the literature. Autoimmun Rev. 2014;13(7):730-5.

49. Tam LS, Chan AY, Chan PK, et al. Increased prevalence of squamous intraepithelial lesions in systemic lupus erythematosus: association with human papillomavirus infection. Arthritis Rheum. 2004;50(11):3619-25.

\section{Publisher's Note}

Springer Nature remains neutral with regard to jurisdictional claims in published maps and institutional affiliations.

\section{Ready to submit your research? Choose BMC and benefit from:}

- fast, convenient online submission

- thorough peer review by experienced researchers in your field

- rapid publication on acceptance

- support for research data, including large and complex data types

- gold Open Access which fosters wider collaboration and increased citations

- maximum visibility for your research: over $100 \mathrm{M}$ website views per year

At $\mathrm{BMC}$, research is always in progress.

Learn more biomedcentral.com/submission 